\title{
eJRIEPS
}

Ejournal de la recherche sur l'intervention en éducation physique et sport

$40 \mid 2017$

Varia

\section{Connaissances et compétences motrices en sport collectif : quoi enseigner?}

Jean-Francis Gréhaigne, Marie-Paule Poggi et Zeineb Zerai

\section{(2) OpenEdition}

\section{Journals}

Édition électronique

URL : https://journals.openedition.org/ejrieps/768

DOI : $10.4000 /$ ejrieps.768

ISSN : 2105-0821

Éditeur

ELLIADD

\section{Référence électronique}

Jean-Francis Gréhaigne, Marie-Paule Poggi et Zeineb Zerai, «Connaissances et compétences motrices en sport collectif : quoi enseigner? ", eJRIEPS [En ligne], 40 | 2017, mis en ligne le 01 janvier 2017, consulté le 28 juin 2022. URL : http://journals.openedition.org/ejrieps/768 ; DOI : https://doi.org/ 10.4000/ejrieps.768

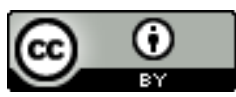

La revue eJRIEPS est mise à disposition selon les termes de la Creative Commons Attribution 4.0 International License. 
eJRIEPS 40 janvier 2017

Connaissances et compétences motrices en sport collectif : quoi enseigner ?

Jean-Francis Gréhaigne*, Marie-Paule Poggi** \& Zeineb Zerai***

* Université de Bourgogne Franche-Comté, France

** Université des Antilles, ESPE de Guadeloupe. CRREF - EA 4538, France

*** ISSEP Ksar Saïd, Université de La Manouba, Tunis, Tunisie

Ce texte en deux parties devaient paraître avec le hors série №1 sport collectif mais comme il n'était pas prêt nous avons du en reculer la publication. Maintenant voilà qui est fait !

\section{Résumé}

Cet article de synthèse s'inscrit dans la rubrique "Innovations objectivées" de la revue eJRIEPS. Son objectif est de présenter et d'analyser les connaissances en sports collectifs qui pourraient être enseignées à l'école et dans une approche compatibles avec l'horaire en lycée et en collège. Une première expérimentation au lycée de Thala en Tunisie a permis de fournir une première indication sur la pertinence des catégories retenues. A la lumière de cette expérimentation, les contenus des programmes français 2008, 2010 et 2016 en EPS sur l'enseignement des sports collectifs sont analysés et examinés en regard des connaissances proposées.

Mots clés : intervention, sport collectif, connaissance, compétence motrice, programme.

En éducation physique et sportive (EPS), les enjeux culturels sont constitués par une conception de la culture sportive en relation avec la notion d'ouverture de l'école sur la vie. Ils visent à répondre à la question : quel rapport l'école entretient-elle avec les pratiques sociales sportives ? Les contenus, construits à partir des pratiques sociales de référence, doivent être réutilisables dans la vie quotidienne dans une perspective d'appropriation culturelle, de dépassement et de progrès social. Dans le bulletin officiel spécial $n^{\circ} 4$ (du 29 avril 2010, p. 1) il était déjà affirmé que par la pratique scolaire, réfléchie, adaptée et diversifiée d'activités physiques, sportives et artistiques, objets du patrimoine et d'une culture contemporaine, l'EPS concourt à l'épanouissement de chaque élève. Elle le confronte aux règles, us, coutumes nécessaires à l'acquisition d'un indispensable savoir " vivre ensemble " respectueux des valeurs de la République, déclinées selon deux 


\section{eJRIEPS 40 janvier 2017}

dimensions.

En sport collectif, il est demandé aux élèves d'être capable de conduire et maîtriser un affrontement individuel ou collectif : exemple pour le niveau 3, gagner le match, mettre en œuvre une organisation offensive qui utilise opportunément la contre-attaque face à une défense qui cherche à récupérer la balle au plus tôt dans le respect des règles (ibid, p. 9). Aussi, les compétences et les connaissances sont au cœur des programmes. Pour les connaissances une définition précise s'impose: Les connaissances renvoient aux informations que doit s'approprier l'élève sur les activités physiques (règlements, évolution, formes sociales de pratiques, etc.), sur son fonctionnement corporel (éléments de physiologie de l'effort, de psychologie, etc.), sur l'activité d'autrui, sur l' environnement. Elles s'énoncent principalement sous forme de principes, de règles, de repères et nécessitent l'utilisation d'un vocabulaire spécifique (Bulletin officiel spécial $n^{\circ} 4$ du 29 avril 2010, p. 3).

Dans un sens étendu et un peu vague, on nomme connaissance les résultats de toute opération intellectuelle : celle-ci est bien une notion aux sens multiples à la fois utilisée dans le langage courant et objet d'étude approfondie chez les chercheurs. Mais, dans une acception plus restreinte et plus précise, la connaissance ne s'applique qu'aux facultés mettant un sujet en rapport avec un ensemble d'idées définies. Les connaissances, leur nature et leurs différentes formes, la manière dont elles sont construites, leur valeur et leur rôle dans les progrès de l'humanité sont étudiés par une diversité de disciplines, comme la didactique, la philosophie, l'épistémologie, la psychologie, les sciences cognitives, l'anthropologie, la sociologie... La didactique d'une discipline comme l'éducation physique et sportive (EPS) est la science qui étudie les phénomènes d'enseignement, les conditions de la transmission de la culture et les conditions de l'acquisition de ces connaissances par un élève. Aussi, dans une réflexion sur les savoirs, les travaux en didactique les considèrent comme des objets vivants, évolutifs et changeants selon les époques et les lieux où on l'utilise (Verret, 1975). Pourtant, la notion de savoir est également peu précise et soulève bien des débats. Par exemple George (1983) parle plutôt de connaissance déclarative, de connaissance procédurale... et de savoir faire. Aussi, avec l'ambition de participer à l'effort de clarification des controverses sur ce thème, cet article tente de faire le point sur ces concepts car informations, connaissances, savoirs dans leurs versants pratiques et théoriques sont souvent employés les uns à la place des autres. A ce propos, Monteil (1985, p. 21) parle "d'ambiguïté sémantique". Il propose trois définitions qui devraient mieux nous aider à mieux caractériser ces notions. Ainsi, l'information est une 


\section{eJRIEPS 40 janvier 2017}

donnée extérieure à la vie du sujet qui peut prendre deux formes liées à son utilisation : soit tirer de l'information d'un ordre préétabli (un livre par exemple) qui renvoie à un processus assez simple ; soit produire un ordre à partir d'informations diverses ce qui renvoie à un processus complexe et permet de construire des connaissances. La connaissance est le résultat de l'expérience personnelle, liée à l'activité du sujet qui assimile l'information et s'y accommode. Dans un système conceptuel, le savoir est la conséquence d'une organisation intellectuelle, où les connaissances mises en réseau par l'activité du sujet, sont conscientes et transmissibles. En ce sens, le savoir s'oppose «à l'ignorance, la foi ou la croyance et est formulable en une proposition dont on admet la vérité pour des raisons intellectuelles et communicables " (Monteil, 1985, p. 24). C'est en fait une véritable connaissance de la connaissance pour cet auteur. Néanmoins, Malglaive (1990) ajoute que d'autres objets interviennent : les savoirs pratiques qui sont directement liés à l'action et à son déploiement, donnent du réel une connaissance partielle mais souvent efficace à l'opérationnalité de l'acte ; les savoir-faire relatifs à la manifestation des aspects moteurs dans l'action matérielle « qui s'agissent plus qu'ils ne se disent ». Le tout pour de Montmollin (1986) renvoyant à des compétences qui constituent des ensembles stabilisés de savoirs et de savoir-faire, de conduites-types, de procédures-standards, de types de raisonnements, que l'on peut mettre en oeuvre sans apprentissage nouveau. En sport collectif aussi, la connaissance des connaissances et des compétences motrices est inséparable de la nécessité d'une pensée complexe pour mieux comprendre qu'au centre de la connaissance est le « joueur connaissant », à la fois observateur, concepteur et réalisateur. Pour Morin (1986) la connaissance proprement humaine est une connaissance fondamentalement liée à la sphère de l'esprit (intelligence + pensée + conscience) car "chacun de ces termes a besoin des autres pour être défini et conçu. Ainsi, la pensée nécessite art et stratégie cognitive, c'est-à-dire de l'intelligence». (p. 199). Au centre de la connaissance, il y a le joueur connaissant qui est, en même temps, un observateur, un concepteur et un acteur de décisions. Cela recouvre chez le joueur une forme d'intelligence tactique qui permet (Gréhaigne \& Godbout, 2014) :

- de simplifier tout en respectant la complexité du jeu ;

- d'analyser l'évolution des configurations du jeu à partir d'indices partiels ;

- de hiérarchiser l'important, le significatif de l'accessoire ;

- de flairer les bons coups et de se servir du hasard de façon favorable.

- d'utiliser la mémoire, l'imagination et l'expérience du joueur. 


\section{eJRIEPS 40 janvier 2017}

Ici, le principe de simplicité (Morin, 1986) impose de réduire les indices pris sur le jeu mais le principe de complexité enjoint de les relier, tout en les distinguant car trop d'indices à prendre en compte tue la vitesse de décision. On peut ajouter que pour Menaut (1993) " la nature de la pensée tactique est d'être organisée et organisante, comme toute forme de connaissance, mais elle est d'être surtout, fondamentalement, transgressante dans l'acte de création » (p. 686). Quant à lui, Fernandez (2016) propose un cadre d'analyse constitué par un couplage cognitif chez les joueurs concevant et décidant de leur activité dans le jeu en relation avec leurs expériences personnelles et des réponses contenues dans le large champ de l'opposition. L'auteur précise que ce cadre ne présente aucune hiérarchie de valeur, d'autant que la situation d'opposition fait primer la réactivité du joueur. Ainsi, l'intelligence en jeu du joueur est le fruit d'une démarche qui le place en situation de lire le jeu et de décider librement comment s'y comporter.

Dans la continuité de cette réflexion, un autre problème mérite d'être considéré. En effet, dans des rapports d'opposition (Delaplace, 1979), l'embarras avec les connaissances et les compétences motrices découle du fait qu'elles sont susceptibles de dégradation avec la fatigue, le stress ou d'une baisse d'attention. Cela se produit également avec un rapport de forces trop déséquilibré où les joueurs d'une équipe sont toujours en retard par rapport à l'évolution du jeu. Ce rapport de forces vécu négativement fait basculer ces joueurs dans un statut de « dominé »... avec ses conséquences sur les possibilités de peser sur le jeu et l'apparente perte de comportements utilisés habituellement. Par exemple, le simple fait de garder la possession de la balle en attaque devient problématique quand l'adversaire est trop largement supérieur et que le principe de "l'égalité des chances à l'inégalité du résultat » (Jeu, 1977, p. 31) ne peut plus être respecté. Le joueur de sport collectif est ainsi le résultat, à cet instant précis, d'une relation dialectique entre le poste qu'il occupe, ses qualités physiques, ses ressources motivationnelles, ses compétences motrices, ses connaissances et l'opposition. Cet état d'équilibre est précaire et sa principale caractéristique est la réversibilité. Un joueur peut revenir à des conduites relevant d'une adaptation à l'affrontement qui sont inférieures à son niveau théorique actuel en fonction d'un rapport de forces vécu défavorablement. Comme en physique, la réversibilité désigne, ici, la propriété de certains systèmes de pouvoir retrouver un état passé par inversion des processus qui ont conduit de l'ancien état à l'état présent. Mais si le trouble cesse ou l'opposition fléchit un peu pour une raison quelconque, le joueur peut retrouver immédiatement son niveau initial. Ces phénomènes constituent un obstacle majeur à toute évaluation des comportements en jeu. 


\section{eJRIEPS 40 janvier 2017}

Néanmoins, concernant les connaissances et les compétences, on voit bien que le problème est loin d'être résolu d'une manière probante et il n'y a donc pas lieu d'en présenter ici une réponse définitive qui serait, de toute façon, sujette à contestation. Le mieux sera d'exposer, en ce qu'elles ont d'essentiel, quelques réponses proposées par les recherches actuelles. Si l'on cherche dans cet article des arguments pour justifier l'idée que les connaissances, la prise de conscience et l'intelligence sont des fardeaux pour le joueur, on sera déçu. En revanche, si on est d'accord pour suivre un questionnement sur les moyens de la connaissance, sur les mises en relation qui lui sont nécessaires, sur son sens, sur ce qui la constitue ainsi que les rapports qu'elle entretient avec l'environnement, alors avançons ensemble sur les chemins de cette connaissance. On pourra, ainsi, tenter de convaincre ceux qui pensent qu'il ne faut pas trop réfléchir pour bien jouer aux sports collectifs.

Pour en revenir à l'EPS et l'école, on y vise des connaissances utiles au traitement de situations particulières et réinvestissables dans des contextes de jeu présentant des similitudes de problèmes à résoudre en vue d'aider à construire un "élève physiquement éduqué ». La principale difficulté vient du fait que les connaissances sont profondément dépendantes voire, parfois, quasiment fusionnées avec les compétences motrices. Cet ensemble constitue un système complexe mais aussi un outil qui doit être disponible sans délai. Ici, l'obstacle provient du fait que le processus est le plus souvent le résultat d'une activité de mise en relation entre plusieurs sources de connaissances et compétences et demande du temps.

Dans cet article de synthèse, nous allons analyser les connaissances en sports collectifs qui devraient être enseignées à l'école et compatibles avec l'horaire en lycée et en collège en relation avec les contenus d'enseignement des sports collectifs des programmes français 2008 et 2010 en EPS.

\section{Les connaissances et compétences en sport collectif}

Tout d'abord, les jeux sont caractérisés par un règlement qui fournit une structure délimitant le problème (cf. aire de jeu, cible, nombre de joueurs, temps accordé). Donc, afin d'identifier un jeu, on parlera de règles primaires (Almond, 1986), de règles constitutives (Cam, Crunelle, Giana, Gorgeorge, \& Labiche, 1979 ; Mérand, 1977), de noyau central de règles (Deleplace, 1966 ; 1979) ou de la logique interne de l'activité (Deleplace, 1979 ; Parlebas, 1991). Ces règles assurent la pérennité du problème posé par le jeu. Ces règles définissent le but du jeu, mais elles concernent surtout les moyens 


\title{
eJRIEPS 40 janvier 2017
}

utilisés pour atteindre cette finalité. Elles interdisent que l'on puisse gagner en employant d'autres moyens que «jouer le jeu ». Ainsi, les règles primaires aident à distinguer les exigences que requiert le jeu et en quoi elles sont spécifiques pour chaque jeu.

En plus de ces règles primaires, il existe des règles secondaires qui proviennent de l'expérience acquise en jeu et qui sont capables d'être changées sans altérer les caractères essentiels du jeu. La pensée tactique devient ici prépondérante car le gain du match nécessite la résolution de problèmes engendrée par l'affrontement (Malho, 1969 ; Deleplace, 1979). Afin de résoudre ces problèmes, des tactiques, des stratégies et des compétences motrices sont utilisées en relation avec les forces et les faiblesses de son équipe, les qualités et les défauts de l'adversaire. Le système des connaissances et des compétences en sport collectif, constituent les règles secondaires (figure 1). Nous avons proposé que celles-ci reposent sur plusieurs grandes catégories (Gréhaigne, 1992 ; Groupe sports collectifs de l'académie de Dijon, 1994 ; Gréhaigne, Zerai, \& Billard, 2014 ; Zerai, 2011) : les règles d'action, les règles de gestion de l'organisation du jeu, les compétences motrices, l'activité perceptive et les règle de gestion du groupe.

\author{
Règles et principes d'action (RA \& PA) \\ Règles individuelles ou collectives pour décider \\ Règles de gestion de l'organisation du jeu (RGOJ) \\ Stratégies et tactiques adoptées \\ Règles de gestion du groupe (RGG) \\ Réseau de compétences, exhortations \\ Compétences motrices et principes moteurs (CM \& PM) \\ Capturer, transporter, propulser le ballon \\ Percevoir, observer des caractéristiques du jeu (PERC) \\ Activité perceptive
}

Figure 1. Catégories d'analyse retenues pour l'approche technologique (Zerai, 2011).

1. 1. Les règles d'action de l'attaque et de la défense (RA \& $P A)$

Pour Vergnaud, Halbwachs et Rouchier, (1978, p. 10) « une règle d'action est une règle qui permet d'engendrer des actions en fonction des valeurs prises par certaines variables de la situation ". Ces variables peuvent évoluer dans le temps, ou d'une situation à l'autre ; une règle (ou une conjonction de règles) n'en est pas moins utilisée pour toute une 


\section{eJRIEPS 40 janvier 2017}

classe de situations. Pour Gréhaigne, Billard, Guillon, et Roche (1989, p. 159), les règles d'action définissent « les conditions à respecter et les éléments à prendre en compte pour que l'action soit efficace». Véritable connaissance sur le jeu, leur utilisation isolée ou articulée avec d'autres règles constitue une réponse à un problème donné. Elles représentent une vérité ponctuelle et certaines règles, vraies temporairement, peuvent s'avérer des obstacles au progrès à d'autres moments (Gréhaigne, 1996). Les règles sont regroupées en principes des actions efficaces sont définis comme une construction théorique et un instrument opératoire qui orientent un certain nombre d'actions dont ils représentent la source et permettent d'agir sur le réel. Ces principes constituent une sorte de cadre de référence macroscopique permettant à l'enseignant d'isoler et de classer les faits qu'il relève et à l'élève de construire des catégories d'un niveau de généralité plus important.

\section{2. Les règles de gestion}

Les « règles de gestion" (Pailhous, 1971) des instruments de connaissance sont des déclarations structurées, qui, dans notre cas, permettent de contraindre, de contrôler et influencer des aspects du jeu. Elles sont utilisées par les joueurs qui, confrontés à une situation problème, vont, pour la résoudre, organiser leurs outils d'une manière plus ou moins efficace pour générer un système de représentation permettant de résoudre le problème rencontré (Menaut, 1998). Ce sont, bien souvent, des propriétés ou des relations essentielles que les joueurs prélèvent dans une situation car il les juge pertinentes pour transformer celle-ci dans le sens souhaité. "Ces invariants opératoires qui structurent l'activité deviennent des concepts pragmatiques au niveau de la représentation " (Samurçay - Pastré, 1995, p. 14). II semble que les règles de gestion de l'organisation du jeu soient des savoirs de même ordre où des connaissances opérationnelles et l'expérience de l'usage que l'on peut en faire ainsi que des effets produits se combinent pour résoudre des suites de configurations du jeu dont l'apparition n'est pas forcément prévisible. Dans le cadre des pratiques physiques sportives et artistiques (PPSA, Bouthier, 1993), les concepts pragmatiques renvoient à l'activité du joueur et à son efficacité dans l'action.

\section{2. 1. Les règles de gestion de l'organisation du jeu (RGOJ)}

La définition préalable de règles de gestion de l'organisation du jeu permet un gain de temps au moment de la décision. Aussi, le joueur a recours aux règles de gestion de l'organisation du jeu afin de résoudre les problèmes en relation avec les forces et les faiblesses de son équipe, les qualités et les défauts de l'adversaire. Celles-ci recouvrent 


\section{eJRIEPS 40 janvier 2017}

un certain nombre de données qui ont trait à la logique de l'activité, à la répartition sur le terrain, à une distribution des rôles, et quelques préceptes simples d'organisation qui peuvent permettre l'élaboration de stratégies et de tactiques. A un niveau plus élaboré, elles comprennent les connaissances sur les configurations prototypiques (Gréhaigne, 2007) l'expérience et les réponses mémorisées par les joueurs à propos de l'affrontement, des circulations du ballon, des situations dangereuses, des opportunités, etc. Ces règles peuvent faire l'objet d'interprétations différentes par les joueurs mais les réponses doivent rester dans une dispersion pas trop large qui ne permettrait pas une conduite et une interprétation collective du jeu. De façon très pratique, elles permettent, aussi, de mettre en relation les éléments pris en compte afin de résoudre les problèmes posés dans la pratique par une situation donnée en vue de répondre dans le jeu aux questions : Qui fait quoi ? Où ? Quand ? Comment ? Pourquoi ?

La pensée tactique reste ici prépondérante car le gain du match nécessite la résolution des situations engendrées par l'affrontement (Menaut, 1993). Afin de résoudre les problèmes posés par le jeu, les règles de gestion de l'organisation du jeu recouvrent un certain nombre de données qui ont trait, aussi, à la logique de l'activité et à des éléments d'organisation constitués au fil du développement de l'activité et de l'expérience des joueurs (Deleplace, 1983).

1. 2. 2. Les règles de gestion du groupe

Comme dans tout groupe, dans chaque équipe s'instaure une distribution des tâches, des conflits ou une répartition tacite des rôles et des fonctions générant ainsi un réseau de compétences (Gréhaigne, Richard, \& Griffin, 2005). À l'interface de la logique du sujet, de la logique du groupe et de la logique du sport considéré, la place du joueur dans ce réseau de compétences constitue, souvent, un révélateur fiable des rapports réciproques entre ce joueur et l'équipe. Au plan général, quand les équipiers se réunissent pour un débat en vue de gagner le match, ils acceptent implicitement de s'influencer les uns les autres et / ou de se laisser influencer (Zerai, 2011). Un joueur qui fait une tentative pour faire valoir son point de vue influence le groupe mais ce qui favorise le travail en groupe, c'est que chacun, en fonction de ses compétences, de ses ressources, de ses motivations puisse influencer le groupe et accepte de se laisser influencer. La cohésion du groupe toutefois ne suppose pas un tout homogène, monolithique ; l'équipe, au contraire, peut comporter un minimum de différenciation entre les joueurs qui la composent. Mais, cette différenciation n'est pas inhérente aux joueurs, elle se base sur une répartition des tâches 


\section{eJRIEPS 40 janvier 2017}

et des fonctions et le groupe n'agit sur son objectif que dans la mesure où il agit sur luimême.

Le débat d'idées (Gréhaigne \& Godbout, 1998 ; Joannisse, Deriaz, \& poussin,1996) constitue alors une pièce centrale d'une conception constructiviste de l'apprentissage des sports collectifs. II consiste après une séquence jouée et le retour d'informations chiffrées en une discussion destinée à faire évoluer ou non le projet d'action de l'équipe en revenant sur la stratégie prévue et en analysant la tactique appliquée. Dans ce type de débat, en explorant collectivement un problème, les joueurs construisent des liens entre les idées de chacun et les problèmes posés par les séquences de jeu. Tout cela vise à l'élaboration de nouvelles réponses adaptées. Cette activité interlocutive peut, néanmoins, révéler des divergences par rapport au jeu mais peut permettre de faire évoluer les représentations, les motifs et les attentes des apprenants. Développer l'appartenance à un groupe par la constitution d'équipes équilibrées entre elles, en partie hétérogènes dans leur constitution et stables dans le temps, est souvent un gage de réussite (Gréhaigne, Billard, \& Laroche, 1999).

1. 3. Compétences motrices et principes moteurs (CM \& PM)

Les élèves ou les joueurs possèdent donc un certain éventail de réponses motrices à leur disposition (celles de la vie courante ou d'autres beaucoup plus spécifiques et élaborées). Les compétences motrices sont constituées d'habiletés qui peuvent être organisées autour de principes moteurs ayant trait à la capture, au transport et à la propulsion de la balle, c'est-à-dire un ensemble de manières d'agir, de gestes ou d'actions coordonnées qui présentent un caractère de rapidité et de stabilité. Nécessairement, ces compétences reposent sur un certain nombre de qualités physiques comme la force ou la vitesse, avec comme fondement théorique, les «principes moteurs" (Gacon, 1984). Ceux-ci soustendent le fonctionnement de ces compétences et sont organisés par :

- le caractère des tensions quand on veut doser un mouvement explosif et moduler ses actions sur la balle ;

- la mise en tension / renvoi de la jambe libre quand on désire lancer loin le ballon (participation de plus en plus de segments à cette action, vers la construction de la chaîne motrice de la frappe de balle) ;

- le placement des chaînes musculaires pour optimiser les réponses motrices.

Les compétences motrices correspondent aussi à la mise en oeuvre, à la coordination et à la régulation, à différents niveaux du contrôle moteur, des signaux envoyés aux muscles en vue de la réalisation du mouvement (Paillard, 1994). Pour les compétences motrices 


\section{eJRIEPS 40 janvier 2017}

dans beaucoup de sports collectifs, un des éléments clé est constituée par la construction du lancer long relativement tendu ainsi que de son attraper en situation d'opposition. La circulation de la balle s'en trouve grandement facilitée avec un agrandissement de l'espace de jeu occupé. De la sorte, un recours à la contre-attaque est plus aisé en permettant d'atteindre des joueurs placés loin à l'avant. Cela permet, également, de lire le jeu différemment, ce qui sera une aide à la constitution d'un répertoire de tactiques individuelles disponibles. Enfin, quand les conditions sont réunies cela permet au joueur en possession du ballon de tirer au but avec une bonne chance de succès et non pas de faire une "passe " au gardien. Tout cet ensemble est à mettre en relation avec la prise en compte des informations qui permettent la gestion et l'exécution de l'échange de balle au travers de la permutation de statut entre passeur et réceptionneur (Mérand, 1977 ; Gréhaigne, 2011).

Dans les apprentissages moteurs, on vise aussi une automatisation partielle des compétences motrices afin de dégager le canal cognitif conscient libéré pour assurer d'autres opérations. Cela consiste, par exemple, à faire exécuter, sauf alerte contraire au niveau de processus infra conscients, un certain nombre de gestes techniques pour offrir la possibilité de faire fonctionner d'autres programmes en même temps. Enfin, d'autres points peuvent perturber le joueur dans sa vitesse d'exécution comme la fatigue, l'importance du match ou une méforme physique passagère ce qui peut entraîner des fautes techniques et des retards dans la résolution des problèmes posés par les configurations du jeu (pour plus de précisions sur ces aspects, on peut consulter Gréhaigne, (Ed), 2016).

1. 4. Percevoir, observer des caractéristiques du jeu (PERC)

Dans le domaine visuel, percevoir suppose de prélever des faits consciemment ou non dans l'environnement, de les ordonner et de les organiser en informations. En sport collectif, du fait de la complexité de l'environnement, les caractéristiques temporelles et spatiales des déplacements et des positions des joueurs ainsi que du ballon sont analysées en fonction du cadre de référence du joueur. La perception est partielle par nature (Ochanine, 1969). Aussi appelle-t-on attention sélective la capacité d'un joueur à sélectionner au sein de l'environnement les indications particulières qui ont un intérêt pour l'exécution de l'action. Pour le joueur, l'expérience et la formation jouent manifestement un rôle de premier plan dans ce processus de reconnaissance des configurations momentanées du jeu. Étant donné la quantité énorme d'informations et le peu de temps dont il dispose pour réagir, la vitesse de sélection devient extrêmement importante. Le 


\section{eJRIEPS 40 janvier 2017}

joueur d'expérience est à même d'assimiler les indications pertinentes fournies par l'environnement du jeu et de former instantanément dans son esprit une image opérative (Ochanine, 1978) qui lui permettra d'agir intelligemment et rapidement face à la situation.

La décision se rapporte au choix des actions-réponses et au moment de leur exécution. Ayant reçu et filtré les informations recueillies sur les configurations du jeu, le joueur doit maintenant décider de l'action appropriée. Là aussi, l'expérience, la mémoire, les connaissances tactiques, de même que le jugement et l'activité mentale ont un impact direct sur la détermination du "quoi faire" et du "quand le faire". De plus, certains facteurs psychologiques tels que la motivation, le plaisir de jouer, la place et le statut dans l'équipe influencent ce processus et ici aussi, la fatigue, le stress peuvent perturber la prise d'informations anticipée et le degré d'élaboration des informations permettant de prédire ou non l'évolution du jeu.

La conceptualisation constitue la clef de voûte des rapports entre pratique et théorie (Vergnaud (2003). Ainsi, toutes les connaissance et compétences analysées précédemment aident à traiter les configurations du jeu de manière efficace et concourent à une prise de décision adaptée à tous les niveaux de l'apprentissage. Ainsi, comprendre les processus de conceptualisation à l'œuvre consistera à considérer à la fois les situations donnant du sens aux concepts, mais aussi à envisager "les systèmes de signifiants langagiers et non langagiers qui permettent de les transformer en concepts explicites " (Vergnaud (1985, p. 247). De ce fait, dans un débat d'idées, les élèves sont invités à établir des actions en projet après confrontation de leurs points de vue. L'interaction verbale permet aux apprenants de choisir une stratégie où la coopération prend tout son sens en vue de construire un plan de jeu en relation avec une juste appréciation des problèmes posés par les confrontations précédentes et leurs rapports d'opposition.

Pour mieux approcher les mécanismes à l'œuvre dans ce genre de fonctionnement, nous allons nous intéresser maintenant à une expérimentation menée en Tunisie avec des filles de lycée qui sont toutes des débutantes dans l'activité handball.

\section{Expérimentation au lycée de Thala}

A partir de ces présupposés théoriques, Zerai (2009) a proposé à des filles un cycle de handball construit sur la base de jeu / débat d'idées / jeu dans une recherche menée avec des élèves de première du lycée de Thala. Le travail s'est étalé sur 12 semaines. A partir de programme tunisien très proche de ceux de la France, les élèves devaient s'organiser 


\section{eJRIEPS 40 janvier 2017}

collectivement et individuellement pour observer, analyser ainsi qu'élaborer des actions en projet en vue de battre une équipe adverse puis d'évaluer et de réguler ce projet. Les débats d'idées ont constitué le fil conducteur pour analyser l'évolution du jeu d'où l'intérêt de l'étude des interlocutions entre élèves lors de ces débats pour suivre l'évolution de la pensée tactique et des registres de fonctionnement. Les verbatim étudiés proviennent donc d'enregistrements effectués pendant ce cycle de 12 semaines, les premiers débats ne procurant que peu de données, les filles restant quasiment muettes ou avec une expression laconique soulignant par là la nécessité d'une expérience minimale du jeu pour pouvoir raisonner sur celui-ci. La construction graduelle de connaissances et de compétences à servi progressivement de référence pour permettre une prise de parole de plus en plus pertinente (Zerai, 2007).

La figure 2 représente la répartition des catégories selon leur apparition dans les verbatims des filles. Ces catégories se répartissent comme suit : $38 \%$ renvoie aux règles de l'organisation du jeu (ROJ). Cette catégorie est constituée par tous les énoncés qui sont en relation avec le jeu, la stratégie et la logique de l'activité. Viennent ensuite avec $19 \%$ les règles de gestion du groupe (RGG). Ces dernières mettent en relief les relations sociocognitives et culturelles, les conflits qui existent entre les filles lors de leurs discussions. Les règles de gestion constituent plus de la moitié des contenus des verbatims (57\%).

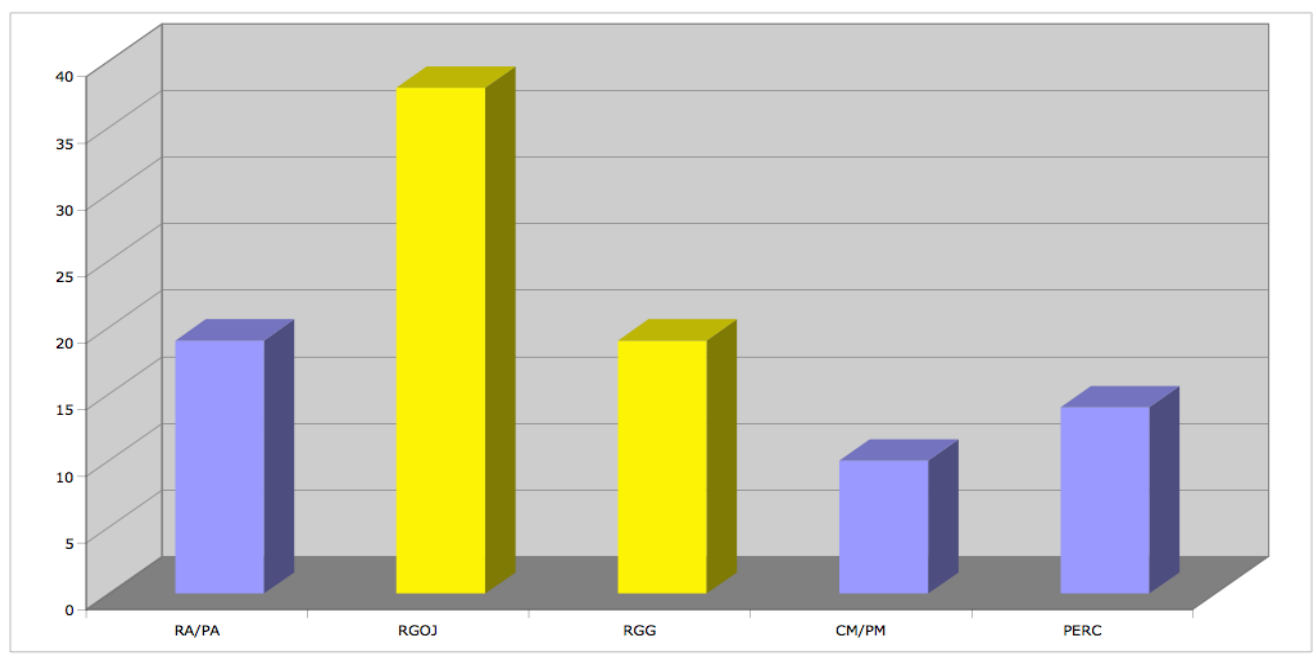

Figure 2. Répartition en pourcentage des catégories dans le verbatim étudié. En jaune les règles de gestion.

Pour les règles et principes d'action (RA/PA), le score est également de $19 \%$. Cette catégorie, comme déjà signalé, met l'accent sur les conditions à respecter et les éléments 


\section{eJRIEPS 40 janvier 2017}

à prendre en compte pour que l'action soit efficace. Les compétences motrices et principes moteurs (CM \& PM) qui sont construits et utilisés par les joueuses permettant, dans le jeu, l'échange, le transport et la propulsion de la balle, représentent $10 \%$ des énoncés dans l'ensemble des verbatim enregistrés. Les propos liés à l'observation et la perception des caractéristiques du jeu sont de 14\%. Cette catégorie traduit les observations pertinentes à propos du jeu pour résoudre un problème rencontré.

Ce travail de Zerai (2011) montre que dans l'analyse qualitative des verbatims des débats d'idées, les interactions verbales permettent l'émergence de facteurs liés au progrès et au développement des compétences tactiques. Ces effets bénéfiques des interactions verbales ont été observés sans qu'aucun conflit entre les filles ait été noté. Ceci a débouché sur une coopération active entre les participantes. Les interactions verbales se caractérisent par un nombre important de tours de parole et une grande richesse dans la dynamique interactive utilisée (Gréhaigne, Zerai, \& Billard, 2014).

Ainsi, chaque catégorie de problèmes permet de concevoir et construire les connaissances à partir de règles et de principes communs, en vue de donner une cohérence aux apprentissages. C'est privilégier des connaissances généralisables qui devraient permettre à tous les élèves quel que soit leur niveau, d'accéder à des connaissances et des compétences motrice en vue d'une culture commune. Néanmoins, cela nécessite de prendre en compte les différences entre les élèves car aider les joueuses à se situer est alors une source de difficultés pour les enseignants. II s'agit là de la construction et l'utilisation de repères précis et concrets, permettant aux élèves d'apprécier leur pratique physique et ce dont ils/elles sont capables. Ceci pose, paradoxalement dans une activité collective, la nécessité de l'individualisation des repères, ceux-ci étant à étalonner de manière propre pour chacune des joueuses.

A partir de ces données nous allons, maintenant, examiner ce que nous proposent les programmes français d'éducation physique et sportive concernant les sports collectifs. L'entrée par la réflexion est y prioritaire car elle permet d'établir des relations de cause à effet entre des résultats et des organisations collectives en vue d'élaborer des hypothèses concernant l'amélioration de l'organisation collective.

\section{Que nous disent les programmes français en EPS ?}

Dans les reformes curriculaires récurrentes en France depuis une dizaine d'années, nous avons choisi de nous appuyer principalement sur le programme de Collège (MEN, 2008) car il nous semble représentatif d'une évolution dans la conception de la didactique des 


\section{eJRIEPS 40 janvier 2017}

sports collectifs. Ce programme propose, au plan temporel, deux niveaux d'exigence : le niveau 1 pour une activité enseignée durant au moins dix heures de pratique effective et le niveau 2 pour une activité enseignée durant au moins vingt heures de pratique effective. Nous avons choisi d'illustrer ce programme avec deux sports collectifs le football et le handball.

En football, dans un jeu collectif à effectif réduit, rechercher le gain du match :

- par des choix pertinents d'actions de passe ou dribble supposant une maîtrise suffisante du ballon pour accéder régulièrement à la zone de marque et tirer en position favorable, face à une défense qui cherche à gêner la progression adverse. S'inscrire dans le cadre d'un projet de jeu simple lié à la progression du ballon. Respecter les partenaires, les adversaires et les décisions de l'arbitre (niveau 1).

- en assurant les montées de balle collectives par une continuité des actions avec et sans ballon. S'inscrire dans le cadre d'un projet de jeu simple lié aux tirs en situation favorable. Observer et co-arbitrer (niveau 2).

En handball, dans un jeu à effectif réduit, rechercher le gain du match :

- par des choix pertinents d'actions de passe ou dribble pour accéder régulièrement à la zone de marque et tirer en position favorable, face à une défense qui cherche à gêner la progression adverse. S'inscrire dans le cadre d'un projet de jeu simple lié à la progression de la balle. Respecter les partenaires, les adversaires et les décisions de l'arbitre (niveau 1).

- en assurant des montées de balle rapides quand la situation est favorable ou en organisant une première circulation de la balle et des joueurs pour mettre un des attaquants en situation favorable de tir quand la défense est replacée. S'inscrire dans le cadre d'un projet de jeu simple lié aux tirs en situation favorable. Observer et co-arbitrer (niveau 2).

Au lycée un référentiel de compétences attendues est établi pour chacune des APSA et est défini selon trois niveaux.

En football, pour gagner le match, mettre en œuvre une organisation offensive :

- qui utilise opportunément la contre-attaque face à une défense qui cherche à récupérer la balle au plus tôt dans le respect des règles (niveau 3 ) ;

- capable de faire évoluer le rapport de force en sa faveur par une occupation permanente de l'espace de jeu (écartement et étagement), face à une défense qui se replie collectivement pour défendre sa cible ou récupérer la balle (niveau 4 );

- capable de faire basculer le rapport de force en sa faveur par un enrichissement 


\section{eJRIEPS 40 janvier 2017}

des alternatives d'attaque (jeu aérien, alternance jeu court/jeu long, renversement, changement de rythme..) face à une défense regroupée (niveau 5).

En hand-ball pour gagner le match, mettre en œuvre une organisation offensive

- qui utilise opportunément la contre-attaque face à une défense qui cherche à récupérer la balle le plus rapidement possible dans le respect des règles (niveau 3 ) ;

- capable de faire évoluer le rapport de forces en sa faveur par l'occupation permanente de l'espace de jeu (écartement et étagement), face à une défense qui se replie collectivement pour défendre sa cible ou récupérer la balle (niveau 4) ;

- capable de faire basculer le rapport de forces en sa faveur par un enrichissement des alternatives d'attaques (s'infiltrer, surpasser, contourner) face à une défense placée (niveau 5).

Dans "une situation d'opposition à effectif réduit ", il y a bien la volonté de mettre en évidence dans un environnement simplifié, les règles de gestion de l'organisation du jeu et d'aller vers des contenus communs en s'installant un peu plus sur le versant transversalité. Avec ce type de texte et un thème d'étude et des connaissances bien identifiées, on pourrait choisir le sport collectif susceptible de faire émerger les comportements visés et on stabiliserait et généraliserait les connaissances et les compétences motrices en utilisant les autres sports collectifs... Le libellé des programmes confronte, également, les enseignants à certaines contradictions. Cogérino (1999) souligne qu'il est précisé que les professeurs doivent évaluer des savoirs " en acte " mais qu'ils doivent aussi sensibiliser les élèves à des règles ce qui relève plutôt de connaissances abstraites voire. La relation dialectique mise en activité / connaissances abstraites est, alors, de même nature que la tension action / parole évoquée pour les situations de verbalisation proposées aux élèves.

La principale caractéristique des récents programmes de 2016, est d'organiser l'éparpillement des connaissances à enseigner sans jamais avoir tiré les enseignements apportés par les précédentes propositions. Pourtant, il est toujours d'actualité que de vrais apprentissages demandent du temps et qu'il est illusoire d'attendre des transformations durables en multipliant les activités des élèves même avec un éclectisme renouvelé. Un programme demande, au moins, trente ans pour donner tous ses effets (Léon, 1980). Alors faisons une vraie pause dans la "course " aux fausses nouveautés et aux pertes de temps dont le «croisement des enseignements » est le summum. Des apprentissages conséquents, évalués en fin de cycle doivent solliciter les connaissances et compétences motrices acquises par les élèves pendant le cycle, permettant ainsi de juger de la capacité 


\section{eJRIEPS 40 janvier 2017}

de ceux-ci à les mobiliser en situation réelle. Ils sont une assurance pour éviter un enseignement de connaissances, de règles ou de principes dévitalisés qui se contenterait de certifier des réponses nouvelles déconnectées de leurs pratiques "culturelles » qui leur donnent du sens et une pertinence.

L'avancée de l'âge aidant, la conceptualisation d'un adulte se fonde toujours, à la fois, sur son expérience et l'évolution de ses connaissances et compétences. Ensemble, elles lui permettent :

- d'identifier des situations comparables là où un autre ne verrait aucune parenté ;

- de puiser dans sa mémoire un cas à peu près semblable ou un ensemble partiel de réponses pour rendre la réalité compréhensible ;

- de construire une solution originale à partir de ses « ressources actualisées ».

Dans la perspective du réinvestissement des règles secondaires en vue d'une vie physique d'adulte de qualité, l'activité authentique de l'apprenant semble un facteur prépondérant pour assurer le succès de l'enseignement et de l'apprentissage en éducation physique et sportive.

\section{Conclusion}

Finalement à l'école, que signifie avoir construit des connaissances et des compétences en sport collectif? Pour l'instant, au lycée ou au collège en éducation physique, nous dirons que l'élève apprend si, confronté à un problème nouveau mais compatible avec les ressources à sa disposition et en relation avec une opposition qu'il peut gérer, il transforme son comportement initial et formule les règles d'action, les RGOJ ou les hypothèses d'action qui l'ont mené à la réussite. Ainsi, on peut penser qu'il a construit des connaissances, des outils d'information et de décision pour débuter dans un autre sport collectif sans être trop naïf et continuer à jouer tout au long de sa vie, les connaissances acquises lui permettant de réguler son activité quand "moins vite, moins haut, moins fort » deviennent d'actualité et que de cheminer vers ses limites amène à des activités physiques raisonnables et raisonnées.

Dans un second article, nous allons approfondir ce que l'enseignement et l'apprentissage des connaissances et des compétences motrices utiles en sport collectif posent comme problème en EPS. 


\section{eJRIEPS 40 janvier 2017}

\section{Bibliographie}

Almond, L. (1986) Primary and secondary rules in games. In R. Thorpe, D. Bunker, \& L. Almond (Eds.), Rethinking games teaching (pp. 73-74). Loughborough, England: Loughborough University of Technology.

Bouthier, D. (1993). L'approche technologique en S.T.A.P.S. : représentations et actions en didactique des A.P.S. Habilitation à diriger des recherches (non publiée) en sciences, spécialité S.T.A.P.S. Université Paris Sud XI.

Cam, Y., Crunelle, J., Giana, E., Gorgeorge, B., \& Labiche, J. (1979). Basket-ball, mémento du CPS FSGT. Paris : Les Éditions Sport et Plein air .

Cogérino, G. (1999). Enseigner des règles de vie au travers de l'EPS. Présentation orale au Colloque de l'AIESEP, Qu'apprennent les élèves en faisant des activités physiques et sportives ? Besançon : avril 1999.

Deleplace, R. (1966) Le rugby. Paris : Colin-Bourrelier.

Deleplace, R. (1979) Rugby de mouvement - Rugby total. Paris : Éducation Physique et Sports.

Deleplace, R. (1983). La recherche sur la spécialisation sportive, l'entraînement, la performance. Actes du colloque la recherche en STAPS (pp. 93-151). Nice. 19-20 Septembre 1983.

De Montmollin, M. (1986). L'intelligence de la tâche : éléments d'ergonomie cognitive. Berne: Peter Lang.

Fernandez, A. (2016). La préréflexion du collectif est-elle représentable dans l'autoconfrontation? https://www.linkedin.com/pulse/w-essai-publi-fernandezfernandez-alain?forceNoSplash=true

Gacon, G. (1984). La course d'endurance. Dijon : CRDP.

George C. (1983). Apprendre par l'action. Paris : Presses Universitaires de France.

Gréhaigne, J.-F. (1992). L'organisation du jeu en football. Paris : ACTIO.

Gréhaigne, J.-F. (1996). Les règles d'action : un support pour les apprentissages. Éducation Physique et Sports, 260, 35-36.

Gréhaigne, J.-F. (Ed.). (2007). Configurations du jeu, débat d'idées et apprentissage des sports collectifs. Besançon : Presses de l'Université de Franche-Comté.

Gréhaigne, J.-F. (Ed.). (2011). Des signes au sens. Le jeu, les indices, les postures et les apprentissages dans les sports collectifs à l'école. Besançon: Presses de l'Université de Franche-Comté. 


\section{eJRIEPS 40 janvier 2017}

Gréhaigne, J.-F. (Ed.). (2016). Les objets de la technique. De la compétence motrice à la tactique individuelle. Besançon : Presses de l'Université de Franche-Comté.

Gréhaigne, J.F., \& Godbout, P. (1998). Observation, critical thinking and transformation : three key elements for a constructivist perspective of the learning process in team sports. . In R. Feingold, R. Rees, G. Barrette, L. Fiorentino, S. Virgilio, \& E. Kowalski (Eds.), Education for life (pp. 109-118). New York : Adelphi University.

Gréhaigne, J.-F., \& Godbout, P. (2014). Dynamic systems theory and team sport coaching Quest, 66 (1), 96-116.

Gréhaigne, J.-F. , Richard, J.-F. , \& Griffin, L. (2005). Teaching and Learning Team Sports and Games. New York: Routledge Falmer.

Gréhaigne, J-F., Zerai, Z., \& Billard, M. (2014). Penées tactiques : des connaissances et compétences pour l'école. In J-F. Gréhaigne (Ed.) L'intelligence tactique. Des perceptions aux décisions tactiques en sports collectifs (pp. 259-278). Besançon : Presses de l'Université de Franche-Comté.

Gréhaigne, J.-F., Billard, M., Guillon, R., \& Roche, J. (1989) Vers une autre conception de l'enseignement des sports collectifs. In G. Bui-Xuan (Ed.) Méthodologie et didactique de l'éducation physique et sportive (pp. 155-172). Clermont-Ferrand : AFRAPS.

Groupe Sports Collectifs de L'Académie de Dijon (1994). Didactique des sports collectifs à l'école. Dossier Éducation Physique et Sport 17.

Jeu, B. (1977). Le sport, l'émotion, l'espace. Paris : Vigot.

Joannisse, R., Deriaz, D., \& Poussin, B. (1996). Expérience d'évaluation formative en éducation physique. Actes du colloque de l'ADMEE, Genève septembre 1994, 213-217.

Léon, A. (1980). Introduction à l'histoire des faits éducatifs. Paris : Presses Universitaires de France.

Malglaive, G. (1990). Enseigner à des adultes. Paris : Presses Universitaires de France. Malho, F. (1969). L'acte tactique en jeu. Paris : Dunod.

Menaut, A. (1998). Le réel et le possible dans la pensée tactique. Contribution à une théorie du jeu sportif. Bordeaux : Presses Universitaires de Talence.

Menaut, A. (1993). A propos d'un type particulier de conduite cognitive: la pensée tactique. Bulletin de psychologie, 412, 684-693.

Mérand, R. (1977). L'éducateur face à la haute performance. Paris : Sport et Plein air. 


\section{eJRIEPS 40 janvier 2017}

Ministère de l'Éducation Nationale. (2008). Bulletin officiel spécial n 6 du 28 août 2008.

Programme de l'enseignement d'éducation physique et sportive pour les classes de sixième, de cinquième, de quatrième et de troisième du collège. http://www.education.gouv.fr/cid22119/mene0817062a.html

Ministère de l'Éducation Nationale. (2010). Bulletin officiel spécial n 4 du 29 avril 2010.

Programme d'enseignement d'éducation physique et sportive pour les lycées d'enseignement général et technologique. http://www.education.gouv.fr/cid51336/mene1007245a.html

Monteil, J.M. (1985). Dynamique sociale et systèmes de formation. Paris : Editions Universitaire, U.N.M.F.R.E.O.

Morin, E. (1986). La Connaissance de la connaissance. Paris : Seuil.

Ochanine, D. (1969). Rôle de l'image opérative dans la saisie du contenu informationnel des signaux. Questions de Psychologie, 4, 209-224.

Ochanine, D. (1978). Le rôle des images opérative dans la régulation des activités de travail. Psychologie et Education, 2(2), 63-72.

Pailhous, J. (1971). Elaboration d'images spatiales et de règles de déplacement. Le travail humain, 34, 299-324.

Paillard, J. (1994). L'intégration sensori-motrice et idéo-motrice. In M. Richelle, J. Requin, \& M. Robert (Eds.), Traité de psychologie expérimentale (pp.927-961). Paris : Presses Universitaires de France.

Parlebas, P. (1991) Didactique et logique interne des APS. Revue EPS, 228, 9-14.

Samurçay, R., \& Pastre, P. (1995). La conceptualisation des situations de travail dans la formation des compétences. Éducation Permanente, 123, 13-31.

Vergnaud, G. (1985). Concepts et schèmes dans une théorie opératoire de la représentation. Psychologie Française, 30, 245-252.

Vergnaud, G. (2003). La conceptualisation clef de voûte des rapports entre pratique et théorie. In DESCO (Ed.) Analyse de pratiques et professionnalité des enseignants (pp. 48-57). CRDP de l'Académie de Versailles.

Vergnaud, G , Halbwachs, F., \& Rouchier, A. (1978). Structure de la matière enseignée, histoire des sciences et développement conceptuel chez l'enfant. Revue Française de Pédagogie, 45, 7-18.

Verret, M. (1975). Le temps des études. Paris : Honoré Champion. 


\section{eJRIEPS 40 janvier 2017}

Zerai, Z., \& Gréhaigne. J-F. (2007). Quelques aspects de la cognition située et distribuée dans l'apprentissage des sports collectifs. Actes du Congrès international Cem 07, 126-133.

Zerai, Z. (2009). Origine culturelle et apprentissage des sports collectifs chez les jeunes filles. Étude comparative d'élèves tunisiennes et françaises. Rapport de recherche (non publié) pour la validation des acquis d'expérience (VAE). IUFM de I'Université de Franche-Comté, Besançon.

Zerai, Z. (2011). Apprentissage du handball chez les jeunes filles Tunisiennes et Françaises; apport de la verbalisation. Thèse de doctorat (non publiée) en Science du Sport. Université de Franche-Comté, Besançon. 